\title{
Custodial symmetry and extensions of the standard model
}

\author{
J. C. Montero* and V. Pleitez ${ }^{\dagger}$ \\ Instituto de Física Teórica, Universidade Estadual Paulista, Rua Pamplona 145, 01405-000, São Paulo, SP-Brazil
}

(Received 11 July 2006; published 21 December 2006)

\begin{abstract}
We show that the extension of the approximate custodial $S U(2)_{L+R}$ global symmetry to all the Yukawa interactions of the standard model Lagrangian implies the introduction of sterile right-handed neutrinos and the seesaw mechanism in this sector. In this framework, the observed quark and lepton masses may be interpreted as an effect of physics beyond the standard model. The mechanism used for breaking this symmetry in the Yukawa sector could be different from the one at work in the vector boson sector. We give three model independent examples of these mechanisms.
\end{abstract}

DOI: 10.1103/PhysRevD.74.115014

PACS numbers: 12.60.-i, 14.60.St, 14.70.Pw

\section{INTRODUCTION}

One of the most important problems in elementary particle physics is concerned about the origin of the fermion masses. Part of the problem, that we will consider here, can be formulated as follows: why do weak-isospin partners have different masses? It is well known that, in the context of the standard model (SM), the weak-isospin partners have different masses because the left- and right-handed components of the fields belong to different representation of the $S U(2)_{L} \otimes U(1)_{Y}$, and they are not related by a symmetry [1]. On the other hand, we may wonder about the natural values for the fermions masses in the context of the SM. For the light quarks $(u, d$ and $s)$ their masses are well below the energy scale, $\Lambda_{\chi} \sim \Lambda_{\mathrm{QCD}}$, of the chiral $S U(3)_{L} \otimes S U(3)_{R}$ symmetry breaking and the spontaneous breakdown of this symmetry dominates the dynamics of these flavors. We can say that their masses are natural in the sense that vanishing them will augment the symmetry of the model. For heavy quarks, whose masses are larger than $\Lambda_{\chi}$, the explicit breaking of the chiral symmetry dominates the dynamics [2]. On the other hand, in the limit of exact global $S U(2)_{L+R}$ isospin symmetry we have that $m_{u}=m_{d}$. The fact that $m_{u} \approx m_{d}$ means that the isospin symmetry is not so badly broken. The case of $S U(3)_{L+R}$ flavor symmetry is not as good realized as the $S U(2)_{L+R}$ but is better than the other higher flavor global chiral symmetries like $S U(4)_{L+R}$ etc. Moreover, from the point of view of the electroweak spontaneous symmetry breaking, we would expect the quark masses to be of the order of magnitude of the symmetry breaking parameter $v=\left(\sqrt{2} / 2 G_{F}\right)^{1 / 2} \sim$ $246 \mathrm{GeV}$. Only the quark top satisfies this requirement since $m_{t} / v \approx 1 / \sqrt{2}$.

In this work we will assume that the natural values for all quarks and leptons are those in which weak-isospin partners have equal masses. This assumption is not in conflict with the discussion about the light and heavy quarks given

\footnotetext{
*Electronic address: montero@ift.unesp.br

${ }^{\dagger}$ Electronic address: vicente@ift.unesp.br
}

above; it is in fact complementary. Hence, we have $M_{u}=$ $M_{d}, M_{l}=M_{\nu_{l}}^{\text {Dirac }}$, where $M_{u}\left(M_{d}\right)$ denotes the mass matrix for the $2 / 3(-1 / 3)$ charged quarks and $M_{l}\left(M_{\nu_{l}}\right)$ denotes the mass matrix for the charged leptons(neutrinos). Notice that at this point the neutrino masses are of Dirac type. If this where the case, the observed mass spectra $m_{u} \neq m_{d}, m_{c} \neq$ $m_{s}$, etc., must be considered already an effect of physics beyond the SM. In the lepton sector whatever is the new physics it must include the seesaw mechanism for generating small masses for the active neutrinos.

Independently of the problem above, it was noted in the late seventies that by ignoring the electromagnetism in the electroweak sector of the SM, $g^{\prime}=0\left(\sin \theta_{W}=0\right)$, the $W$ boson fields $W^{ \pm}, W^{3} \equiv Z$ transform as triplet under a global $S U(2)_{L+R}$ symmetry and, as a consequence, the masses of the three vector bosons are equal: $M_{Z}=M_{W^{ \pm}}$ [3]. The $\rho$-parameter defined as $\rho \equiv M_{W}^{2} / M_{Z}^{2} \cos ^{2} \theta_{W}$ has in this limit $\left(\cos \theta_{W}=1\right)$ the value $\rho=1$. The inclusion of electromagnetism through the $U(1)_{Y}$ factor i. e., $g^{\prime} \neq 0$ (or $\sin \theta_{W} \neq 0$ ), breaks the global symmetry and the $W^{3}-B$ mixing introduces the zero order (in the sense that it does not depend neither on $g$, nor on $g^{\prime}$ ) correction factor $\cos ^{2} \theta_{W} \neq 1$ to the ratio of the vector boson masses but still we have $\rho=1$ at the tree level. When radiative corrections are considered $\rho \approx 1$. That global symmetry can be in some cases identified with the $S U(2)$ isospin, and since it protects the value of $\rho$ from radiative corrections it is called custodial symmetry. This sort of symmetry has been also explored in other contexts [4,5].

Let us review how the $S U(2)$ custodial symmetry has been implemented in the scalar sector of the SM. This model has only one Higgs scalar doublet $H=\left(h^{+} h^{0}\right)^{T}$ transforming as $(2,+1)$ under $S U(2)_{L} \otimes U(1)_{Y}$. By introducing the 2-doublet [6]

$$
\Phi=\frac{1}{\sqrt{2}}(\tilde{H} H)
$$

where $\tilde{H}=\epsilon H^{*}$, we can write the usual $S U(2)_{L} \otimes U(1)_{Y}$ invariant Lagrangian of the Higgs scalar sector in terms of the 2-doublet 


$$
\mathcal{L}=\operatorname{Tr}\left[\left(D_{\mu} \Phi\right)^{\dagger} D^{\mu} \Phi\right]-V\left(\Phi^{\dagger} \Phi\right),
$$

where $V\left(\Phi^{\dagger} \Phi\right)=-\mu^{2} \operatorname{Tr} \Phi^{\dagger} \Phi+\lambda\left(\operatorname{Tr} \Phi^{\dagger} \Phi\right)^{2}$ and the covariant derivative becomes

$$
D_{\mu} \Phi=\left(\partial_{\mu} \Phi+i \frac{g}{2} \vec{W}_{\mu} \cdot \vec{\sigma} \Phi+i \frac{g^{\prime}}{2} B_{\mu} \Phi \sigma_{3}\right) .
$$

We see that the Lagrangian in Eq. (2) is invariant under the local and global $S U(2)_{L} \otimes U(1)_{Y}$ symmetry: $\Phi \rightarrow L \Phi$ and $\Phi \rightarrow \Phi \exp \left(-i \sigma_{3} / 2\right)$. However in the limit when $g^{\prime}=$ 0 we have a larger right-handed symmetry: $\Phi \rightarrow \Phi R^{\dagger}$ where $R$ is a global $S U(2)_{R}$ transformation. In this case we have that $\Phi \sim(\mathbf{2}, \mathbf{2})$ under global $S U(2)_{L} \otimes S U(2)_{R}$. The Lagrangian in Eq. (2) has an approximate (valid when $\left.g^{\prime}=0\right) S U(2)_{L} \otimes S U(2)_{R}$ global symmetry which is broken to $S U(2)_{L+R}$ when the neutral component of $H$ gains a vacuum expectation value, $\langle\Phi\rangle=\operatorname{diag}(v, v) / \sqrt{2}$. Now $L=R$ and $\langle\Phi\rangle \rightarrow L\langle\Phi\rangle L^{\dagger}$ under $S U(2)_{L+R}$. Next, $S U(2)_{L+R}$ is broken when $g^{\prime} \neq 0$.

In the next section, motivated by the idea that it may be natural that, in the context of pure SM, the weak-isospin partners have equal masses, we will extend the custodial symmetry to all the Yukawa sectors of the SM. Within the minimal SM representation content, i. e., no sterile neutrinos at all, that symmetry can be implemented with the known fermions only in the quark sector. Quarks are strong interacting particles so that it makes sense to consider the turning off of the electromagnetic interactions. Moreover, by adding right-handed neutrinos we are able to extend this approximate custodial symmetry also to the Yukawa lepton sector. In this sector we may consider that the equivalent to the "strong interactions", with respect to which we can turn off the electromagnetic interactions, are the large Majorana masses of the right-handed neutrinos. The latter ones are sterile with respect to the SM interactions, and trigger the type-I seesaw mechanism [7]. Hence, this mechanism is mandatory in the scheme.

\section{THE CUSTODIAL SYMMETRY IN THE YUKAWA SECTOR}

The condition under which isospin partners like proton and neutron have the same mass was put forward by Weinberg in the early seventies in the context of an $S U(2)_{L} \otimes S U(2)_{R} \otimes U(1)$ model [8]. This is straightforward extended for $u$ and $d$ quarks. The generalization of this symmetry to the other weak-isospin doublets implies that this equality is valid for any $2 / 3$ and $-1 / 3$ charged quarks and similarly in the lepton sector. As we said in the previous section the extension of the custodial symmetry to the Yukawa sector, as it was suggested recently in another context [9], implies that the weak-isospin partners have the same mass. On the other hand, in order to be able to implement this symmetry in the Yukawa leptonic sector we ought to introduce right-handed neutrinos. If we consider that the weak-isospin partners have equal masses the observed fermion masses should be already seen as an effect of new interactions that break explicitly, or not, the custodial symmetry. We give some model independent examples of possible new physics breaking the custodial symmetry and producing the observed fermion masses. They are: (i) the existence of extra fermions transforming as singlets under local $S U(2)_{L}$, (ii) several scalar doublets, and/or, (iii) new neutral vector bosons with nonuniversal couplings to fermions.

As usual we define the minimal representation content in the standard model plus right-handed neutrinos: the doublets $L_{a L}=\left(\nu_{a L} l_{a L}\right)^{T} \sim(\mathbf{2},-1), \quad Q_{i L}=\left(u_{i L} d_{i L}\right)^{T} \sim$ $(\mathbf{2}, 1 / 3)$, and the singlets $l_{a R} \sim(\mathbf{1},-2), u_{i R} \sim(\mathbf{1}, 4 / 3)$, $d_{i R} \sim(\mathbf{1},-2 / 3)$, and $\nu_{a R} \sim(\mathbf{1}, 0)$, where $a=e, \mu, \tau$ and $i=1,2,3$. Notice that it is only because we have included right-handed neutrinos that we are able to define in the lepton sector the 2-doublets, $(\mathbf{2}, \mathbf{2})$ under $S U(2)_{L} \otimes$ $S U(2)_{R}, \mathrm{~L}_{a b}=\left(\bar{L}_{a_{L}} \nu_{b R} \bar{L}_{a L} l_{b R}\right)$. In the quark sector however we already have all the fields needed to write the 2doublets $\mathrm{Q}_{i j}=\left(\bar{Q}_{i L} u_{j R} \bar{Q}_{i L} d_{j R}\right)$. Both 2-doublets $\mathrm{L}_{a b}$ and $\mathrm{Q}_{i j}$ transform under $S U(2)_{L} \otimes S U(2)_{R}$ as $\Phi^{\dagger}$ in the last section. Thus, we can write the Yukawa interactions which are invariant under this global symmetry as follows:

$$
-\mathcal{L}_{Y}^{\prime}=h_{a b} \operatorname{Tr}\left(\mathrm{L}_{a b} \Phi\right)+g_{i j} \operatorname{Tr}\left(\mathrm{Q}_{i j} \Phi\right)+\text { H.c. }
$$

We note from the Yukawa interactions in Eq. (4) that if the custodial symmetry is not broken the mass of the charged lepton in each generation is equal to the mass of the respective neutrino. The same happens in the quark sector: the $u$-type quarks have the same mass of the $d$-type quarks, for each generation. Explicitly we have $\left(M_{l}\right)_{a b}=$ $\left(M_{\nu_{l}}^{\text {Dirac }}\right)_{a b}=h_{a b} v / \sqrt{2}$; and $\left(M_{u}\right)_{i j}=\left(M_{d}\right)_{i j}=g_{i j} v / \sqrt{2}$. After diagonalizing these matrices we obtain the unrealistic mass relations $\hat{M}_{l}=\hat{M}_{\nu_{l}}^{\text {Dirac }}=(v / \sqrt{2}) \operatorname{diag}\left(h_{e}, h_{\mu}, h_{\tau}\right)$; and $\hat{M}_{u}=\hat{M}_{d}=(v / \sqrt{2}) \operatorname{diag}\left(g_{1}, g_{2}, g_{3}\right)$. It is interesting that in $S U(2)_{L} \otimes S U(2)_{R} \otimes U(1)$ gauge models the custodial symmetry implies relations between the masses of quarks and leptons of each generation [1].

Thus, we have to break the custodial symmetry in order to generate the observed fermion masses. In the context of the minimal SM i. e., no sterile neutrinos at all, this is not a problem because there is no a custodial symmetry in the lepton sector since, as we said before, there is no enough fields to define 2-doublets as the $\mathrm{L}_{a b}$ above. The custodial symmetry in the boson sector is broken by turning on the electromagnetic interactions i. e., $g^{\prime} \neq 0$, and also by the Yukawa interactions, with or without right-handed neutrinos, if we write them as

$$
\begin{aligned}
-\mathcal{L}_{Y}= & \left(\bar{Q}_{i L} \Gamma_{i j}^{u} u_{j R}+\bar{L}_{a L} \Gamma_{a b}^{\nu} \nu_{b R}\right) \tilde{H}+\left(\bar{Q}_{i L} \Gamma_{i j}^{d} d_{j R}\right. \\
& \left.+\bar{L}_{a L} \Gamma_{a b}^{l} l_{b R}\right) H+\text { H.c., }
\end{aligned}
$$

where $i, j=1,2,3 ; a, b=e, \mu, \tau$ and we have here, and below, omitted summation symbols. If all Yukawa couplings $\Gamma$ s in Eq. (5) are different, the generated Dirac 
masses in each charged sector are different and arbitrary. The breaking mechanism of the custodial symmetry in Eq. (5) is the same as in the boson sector: $g^{\prime} \neq 0$.

However, instead of assuming the interactions in Eq. (5), we can look for different ways to obtain arbitrary masses and mixing matrices, in all charged sectors, starting from Eq. (4). Then, we will look for mechanisms that can produce realistic masses for all fermions in the standard model by breaking the custodial symmetry in at least three model independent examples: (i) a generalized seesaw mechanism [10,11]; (ii) multi-Higgs doubletlike SM extensions; and (iii) radiative corrections mechanism. The latter case involves extra neutral vector bosons with nonuniversal couplings to leptons. In all these cases the seesaw mechanism is necessary in the neutrino sector.

\section{A. Generalized seesaw mechanism}

One way to obtain a general mass matrix for quarks and leptons is to add at least one extra $u$ - and $d$-type quarks, $e^{-}$-type charged leptons, and three sterile neutrinos $\nu_{R}$. These fields transform under $S U(3)_{C} \otimes S U(2)_{L} \otimes U(1)_{Y}$ as: $\quad U_{L, R} \sim(\mathbf{3}, \mathbf{1}, 4 / 3), \quad D_{L, R} \sim(\mathbf{3}, \mathbf{1},-2 / 3), \quad E_{L, R} \sim$ $(\mathbf{1}, \mathbf{1},-2)$, and $\nu_{R} \sim(\mathbf{1}, \mathbf{1}, 0)$, respectively. In this way the Yukawa interactions to be added to Eq. (4), for breaking explicitly the custodial symmetry, are

$$
\begin{aligned}
-\mathcal{L}_{Y}= & \bar{Q}_{i L} g_{i U} U_{R} \tilde{H}+\bar{Q}_{i L} g_{i D} D_{R} H+\bar{U}_{L} M_{U i} u_{i R} \\
& +\bar{D}_{L} M_{D i} d_{i R}+M_{U U} \bar{U}_{L} U_{R}+M_{D D} \bar{D}_{L} D_{R} \\
& +\bar{L}_{a L} h_{a E} E_{R} H+M_{E E} \bar{E}_{L} E_{R} \\
& +\frac{1}{2} \overline{\left(\nu_{a R}\right)^{c}}\left(M_{R}\right)_{a b} \nu_{b R}+\text { H.c., }
\end{aligned}
$$

where $i, j=1,2,3 ; a, b=e, \mu, \tau$. The Yukawa coupling matrices in the $u$-like quarks and charged leptons are, respectively,

$$
\begin{gathered}
\Gamma_{u}=\left(\begin{array}{cccc}
g_{11} & g_{12} & g_{13} & g_{1 U} \\
g_{21} & g_{22} & g_{23} & g_{2 U} \\
g_{31} & g_{32} & g_{33} & g_{3 U} \\
\frac{\sqrt{2}}{v} M_{U 1} & \frac{\sqrt{2}}{v} M_{U 2} & \frac{\sqrt{2}}{v} M_{U 3} & \frac{\sqrt{2}}{v} M_{U U}
\end{array}\right), \\
\Gamma_{l}=\left(\begin{array}{cccc}
h_{e e} & h_{e \mu} & h_{e \tau} & h_{e E} \\
h_{\mu e} & h_{\mu \mu} & h_{\mu \tau} & h_{\mu E} \\
h_{\tau e} & h_{\tau \mu} & h_{\tau \tau} & h_{\tau E} \\
\frac{\sqrt{2}}{v} M_{E e} & \frac{\sqrt{2}}{v} M_{E \mu} & \frac{\sqrt{2}}{v} M_{E \tau} & \frac{\sqrt{2}}{v} M_{E E}
\end{array}\right),
\end{gathered}
$$

and similarly, the $d$-like quark Yukawa matrix is obtained by replacing in $\Gamma_{u}, g_{i U} \rightarrow g_{i D}, M_{U i} \rightarrow M_{D i}$ and $M_{U U} \rightarrow$ $M_{D D}$. The related mass matrices can implement a seesawlike mechanism if $M_{U U} \gg g_{i j} v / \sqrt{2}, g_{i U} v / \sqrt{2}, M_{U i}$, and similar conditions in the other charged sectors. In fact, a particular case of the Yukawa matrix for the charged leptons $\Gamma_{l}$, where the submatrix $h_{a b}$ is antisymmetric, was worked out in Ref. [11] showing that it is possible to generate the observed charged lepton masses, with a fine tuning which is not worst than the usual one in the SM. In the neutrino sector we have the $6 \times 6$ mass matrix

$$
-\mathcal{M}_{\nu}=\frac{1}{2}\left(\begin{array}{cc}
0 & \frac{v}{\sqrt{2}} h \\
\frac{v}{\sqrt{2}} h^{T} & M_{R}
\end{array}\right),
$$

where $M_{R}$ is a general $3 \times 3$ matrix. With this scheme we obtain a general mixing between left-handed neutrinos which allows an arbitrary mixing matrix in the charged currents coupled to $W^{ \pm}$. We can verify also that the neutrino masses compatible with neutrino oscillation data implies the existence of at least two large Majorana mass scales. Assuming for simplicity that $(v / \sqrt{2}) h \rightarrow$ $(v / \sqrt{2}) \operatorname{diag}\left(h_{e}, h_{\mu}, h_{\tau}\right) \approx \operatorname{diag}\left(m_{e}, m_{\mu}, m_{\tau}\right)$, and $M_{R}=$ $\operatorname{diag}\left(M_{1}, M_{2}, M_{3}\right)$, we obtain $M_{1} \sim M_{2}=10^{9} \mathrm{GeV}$ and $M_{3}=10^{12} \mathrm{GeV}$.

In this case the mixing matrices in the quark and lepton sectors are not exactly unitary and there is also flavor changing neutral currents (FCNC) coupled to the $Z^{0}$ and the neutral scalars. However, these sort of currents are naturally suppressed by the mass of these extra fermions. For a general analysis see Refs. [12]. On the other hand, the value of the $\rho$ parameter is still protected from radiative corrections since the charged fermion singlets do not contribute to $\rho$ because they couple to $Z^{0}$ only through vector currents. We have verified, using the general expressions given by Veltman [13], that $\left.\delta \rho\right|_{\text {singlets }}=0$, at least at the one loop level. Anyway, this issue has to be considered in a particular model in which the $S U(2)_{L} \otimes U(1)_{Y}$ symmetry and the extra fermions may be imbedded [14,15].

\section{B. Multi-Higgs doubletlike extensions of the standard model}

Another mechanism for breaking the custodial $S U(2)_{L+R}$ symmetry is implemented by adding extra scalar doublets to the SM. Let us suppose that we add four Higgs doublets $H_{i}=\left(h_{i}^{+}, h_{i}^{0}\right)^{T}, i=2,4$ and $H_{j}=\left(h_{j}^{0},-h_{j}^{-}\right)^{T}$, $j=1,3$, allowing to define the 2-doublets $\Phi_{12}=$ $\left(H_{1}, H_{2}\right) / \sqrt{2}$, and $\Phi_{34}=\left(H_{3}, H_{4}\right) / \sqrt{2}$. Other $\left(H_{i}, H_{j}\right)$ combinations may be avoided by imposing appropriate discrete symmetries. In this case we have

$$
\begin{aligned}
-\mathcal{L}_{Y}^{\prime \prime}= & h_{a b} \operatorname{Tr}\left(\mathrm{L}_{a b} \Phi_{12}\right)+h_{a b}^{\prime} \operatorname{Tr}\left(\mathrm{L}_{a b} \Phi_{34}\right) \\
& +g_{i j} \operatorname{Tr}\left(\mathrm{Q}_{i j} \Phi_{12}\right)+g_{i j}^{\prime} \operatorname{Tr}\left(\mathrm{Q}_{i j} \Phi_{34}\right)+\text { H.c. }
\end{aligned}
$$

Denoting $\left\langle h_{i}^{0}\right\rangle=v_{i} / \sqrt{2}$ and considering $v_{i} \neq v_{j}$, for $i \neq$ $j$, we break the custodial symmetry, and obtain the mass matrices

$$
\begin{aligned}
\left(M_{l}\right)_{a b} & =\frac{1}{\sqrt{2}}\left[h \boldsymbol{v}_{2}+h^{\prime} \boldsymbol{v}_{4}\right]_{a b}, \\
\left(M_{\nu_{l}}^{\text {Dirac }}\right)_{a b} & =\frac{1}{\sqrt{2}}\left[h v_{1}+h^{\prime} v_{3}\right]_{a b},
\end{aligned}
$$




$$
\begin{aligned}
& \left(M_{d}\right)_{i j}=\frac{1}{\sqrt{2}}\left[g v_{2}+g^{\prime} v_{4}\right]_{i j}, \\
& \left(M_{u}\right)_{i j}=\frac{1}{\sqrt{2}}\left[g v_{1}+g^{\prime} v_{3}\right]_{i j},
\end{aligned}
$$

where we are assuming real vacuum expectation values for simplicity.

The point here is that because all the vacuum expectation values are different from each other, the mass matrices $M_{l}$ and $M_{\nu_{l}}^{\text {Dirac }}$ are numerically different so that they can be diagonalized by quite different unitary matrices. This sort of models have FCNC which implies fine tuning in some of the couplings since the Higgs scalar can not be arbitrarily heavy. A more economical model with natural FCNC suppression [16] is the one in which one of the two 2doublets, $\Phi_{12}$, couples only to leptons, and the other, $\Phi_{34}$, couples only to quarks. Then $M_{l}=h v_{2} / \sqrt{2}, M_{\nu_{l}}^{\text {Dirac }}=$ $h v_{1} / \sqrt{2}, M_{d}=g^{\prime} v_{4} / \sqrt{2}$, and $M_{u}=g^{\prime} v_{3} / \sqrt{2}$. In this case the Dirac masses of the neutrinos are different from the charged lepton masses and, if we assume that the Majorana mass matrix for the right-handed neutrinos $M_{R}$ is already diagonal, there is no a realistic mixing in the leptonic sector. However, if $M_{R}$ is not diagonal the active neutrino mass matrix is $M_{\text {active }}^{\nu} \approx-M_{\nu_{l}}^{\text {Dirac }} M_{R}^{-1}\left(M_{\nu_{l}}^{\text {Dirac }}\right)^{T}$, which is numerically different from $M_{\nu_{l}}^{\text {Dirac }}$, and realistic mixing in the lepton sector may arise. But, if even in this case the mixing is not the required one, we can still apply the mechanism of radiative corrections, discussed in the next subsection, in order to generate the appropriate mixing matrix in the lepton sector and we will get $\tilde{M}_{\text {active }}^{\nu} \approx$ $-\tilde{M}_{\nu_{l}}^{\text {Dirac }} M_{R}^{-1}\left(\tilde{M}_{\nu_{l}}^{\text {Dirac }}\right)^{T}$ (see below).

\section{Radiative correction breakdown of the custodial symmetry}

Here we will assume again that the Yukawa interactions at the tree level are those in Eq. (4), i. e., $\left(\Gamma_{l}^{\prime}\right)_{a b}=\left(\Gamma_{\nu}^{\prime}\right)_{a b} \equiv$ $h_{a b},\left(\Gamma_{u}^{\prime}\right)_{i j}=\left(\Gamma_{d}^{\prime}\right)_{i j} \equiv g_{i j}$. Next, let us assume that the correct quark masses have already been obtained by some of the two mechanisms considered above but lepton weak-isospin partners are still mass degenerate. Then, we have to break only the degeneracy between the charged lepton and the Dirac neutrino masses in order to have an appropriate mixing in this sector. The question is that, if the Majorana mass matrix for right-handed neutrinos is assumed diagonal, the charged lepton and neutrino mass matrices are diagonalized by the same unitary matrices and, as discussed above, in this case there is no mixing in the leptonic sector $U_{\mathrm{PMNS}}=U_{l L}^{\dagger} U_{\nu L}$. But in the present case since $M_{l}=M_{\nu_{l}}^{\text {Dirac }}$ then $U_{l L}=U_{\nu L}$, and thus we may have no enough mixing among active neutrinos. Thus, we have to break the mass degeneracy, or induce more corrections to the lepton masses, in order to have an appropriate mixing matrix. The small masses for the active neutrinos will arise through the seesaw mechanism as in the other cases. In this case the custodial symmetry break- down of the SM can be implemented by radiative corrections involving fields that are not present in this model.

A realization of this mechanism implies the existence of extra neutral vector bosons, say $Z^{\prime}$, with nonuniversal couplings with right-handed leptons, which can induce a general finite mass contribution for these particles. This sort of models already exist in literature, see for instance Ref. [17]. The extra neutral vector boson couples to active left-handed neutrino only through the mixing of $Z$ with $Z^{\prime}$ which may arise at the tree level or at higher orders. This is the case of models in which left-handed and right-handed neutrino components transform in different representation with respect to the symmetry that include the $Z^{\prime}$. The most simple case is an extra $U(1)$ symmetry under which only the right-handed neutrinos are active. The calculability of the masses is used in the sense of Refs. [8,18]. In this case there are diagrams, like the one in Fig. 1, that generate finite mass contributions for neutrinos and similarly for charged leptons. In the figure the tadpole denotes $\left\langle h^{0}\right\rangle=$ $v / \sqrt{2}$. We need here only that $Z^{\prime}$ has nonuniversal couplings with right-handed leptons, $f_{R}^{l}, f_{R}^{\nu}$. Contributions to the matrix $h_{a b}$, arising from Fig. 1, are proportional (up to logarithm factors) to $\mathrm{g}_{a L}^{\nu} f_{b R}^{v} M_{\nu_{l}}^{\text {Dirac }} \Delta$, where $\Delta \equiv$ $\left(v^{2} / \sqrt{2}\right) /\left(M_{Z}^{2}+M_{Z^{\prime}}^{2}\right)$ and $\left(M_{\nu_{l}}^{\text {Dirac }}\right)_{a b}=h_{a b} v / \sqrt{2}$. On the other hand, contributions to the mass matrix of the charged leptons are proportional to $\mathrm{g}_{a L}^{l} f_{b R}^{l} M_{\nu_{l}}^{\text {Dirac }} \Delta$. If only righthanded neutrinos and the Higgs scalar carry the charge related to $Z^{\prime}$, the diagram in Fig. 1 is the only one in the language in which the gauge symmetry breaking is introduced explicitly by tadpoles [18]. In this way we can generate arbitrary contributions for the masses of both charged lepton sectors.

Then, in the lepton sector the mass matrices, after taken into account the radiative corrections, become

$$
\begin{aligned}
\left(\tilde{M}_{l}\right)_{a b} & \approx\left(M_{\nu_{l}}^{\text {Dirac }}\right)_{a b}+\left(2 s_{W}^{2}-1\right)\left(M_{\nu_{l}}^{\text {Dirac }}\right)_{a c}\left(f_{R}^{l}\right)_{c b} \Delta, \\
\left(\tilde{M}_{\nu_{l}}^{\text {Dirac }}\right)_{a b} & \approx\left(M_{\nu_{l}}^{\text {Dirac }}\right)_{a b}+\left(M_{\nu_{l}}^{\text {Dirac }}\right)_{a c}\left(f_{R}^{\nu}\right)_{c b} \Delta,
\end{aligned}
$$

where $f_{L}^{l}$ and $f_{R}^{\nu}$ are general, model dependent, nondiagonal matrices. Since the mixing of $Z$ with heavier $Z^{\prime}, \ldots$ has to be small, say $\Delta \lesssim O\left(10^{-3}\right)[19], \tilde{M}_{l}$ and $\tilde{M}_{\nu_{l}}^{\text {Dirac }}$ are

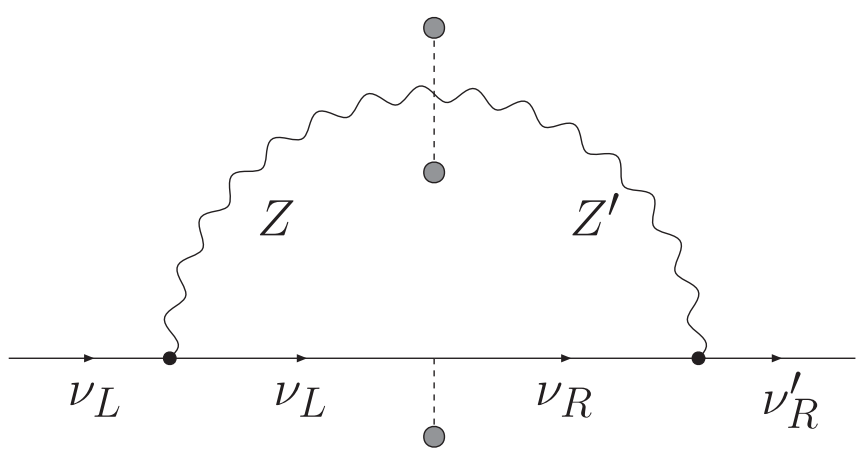

FIG. 1. Possible finite contributions for the neutrino Dirac masses. Similar diagrams do exist for charged leptons. 
still of the same order of magnitude but numerically they can differ enough, depending of the values of $f_{R}^{l}, f_{R}^{\nu}$, to be diagonalized by different unitary matrices in such a way that the mixing matrix in the charged currents coupled to the boson $W$ does appear. The fact that $\tilde{M}_{l} \approx \tilde{M}_{\nu_{l}}^{\text {Dirac is not a }}$ problem because now we can implement the seesaw mechanism and the total neutrino mass matrix has the form as in Eq. (8). This mechanism is useful for generating a realistic mixing in the lepton sector when the 2 Higgs doublets extension of the SM with no FCNC is considered as in the previous subsection. In this case, although the masses of neutrinos and charged leptons are numerically different, both mass matrices are diagonalized by the same unitary matrices since $M_{l}=M_{\nu_{l}}^{\text {Dirac }}\left(v_{2} / v_{1}\right)$. When radiative corrections are considered, the latter relation is not valid anymore.

In general, in models with extra neutral vector bosons with nonuniversal interactions there are also FCNC that impose constraints on the parameters of the models, see for instance Refs. [19,20].

\section{CONCLUSIONS}

We have seen that if we want to extend the custodial symmetry to the Yukawa sector in the standard model it is mandatory to introduce right-handed neutrinos and hence the seesaw mechanism. We have considered three different model independent ways, involving new physics, to break that symmetry, generating the appropriate masses in the fermion sector. Our analysis was model independent but all these mechanisms may be implemented in a particular model in which the custodial symmetry is imposed from the beginning. The radiative correction mechanism may be implemented with scalar neutral bosons or heavy leptons as well. In the latter case there are more than one tadpole in the internal fermion line and the diagram is finite.

In fact, because the measured value of $\rho$ is, experimentally, near 1 , we know that the custodial symmetry of the $\mathrm{SM}$ is not so badly broken. But, if this is an approximate symmetry of the full model, its breakdown in the fermion and in the boson sectors may be related to different sources (mechanisms) as we have shown in this work. In all these cases the seesaw mechanism is mandatory in the neutrino sector in order to generate small masses for the active neutrinos.

\section{ACKNOWLEDGMENTS}

This work was partially supported by CNPq under the processes 305185/03-9 (J. C. M.) and 306087/88-0 (V. P.).
[1] R. R. Volkas, Phys. Rev. D 53, 2681 (1996).

[2] A. V. Manohar and C. T. Sachradja, Phys. Lett. B 592, 473 (2004).

[3] M. Veltman, Nucl. Phys. 123, 89 (1977); Acta Phys. Pol. B 8, 475 (1977).

[4] P. Sikivie, L. Susskind, M. Voloshin, and V. Zakharov, Nucl. Phys. B173, 189 (1980); R. S. Chivukula, M. J. Dugan, M. Golden, and E.H. Simmons, Annu. Rev. Nucl. Part. Sci. 45, 255 (1995).

[5] K. Agashe, R. Contino, L. Da Rold, and A. Pomarol, Phys. Lett. B 641, 62 (2006); M. Carena, E. Pontón, J. Santiago, and C. E. M. Wagner, Nucl. Phys. B759, 202 (2006).

[6] S. Willenbrock, hep-ph/0410370.

[7] M. Gell-Mann, P. Ramond, and R. Slansky, in Supergravity, edited by P. van Niewenhuizen and D.Z. Freedman (North-Holland, Amsterdam, 1979), p. 315; T. Yanaguida, in Proceedings of the Workshop on Unified Theory and Baryon Number in the Universe, edited by O. Sawada and A. Sugamoto (KEK, Tsukuba, 1979); R. N. Mohapatra and G. Senjanovic, Phys. Rev. Lett. 44, 912 (1980).

[8] S. Weinberg, Phys. Rev. Lett. 29, 388 (1972).

[9] A. G. Dias, J. C. Montero, and V. Pleitez, Phys. Rev. D 73, 113004 (2006); Phys. Rev. D 73, 113004 (2006).

[10] A. Davidson and K. Wali, Phys. Rev. Lett. 59, 393 (1987).
[11] J. C. Montero, C. A. de S. Pires, and V. Pleitez, Phys. Rev. D 65, 093017 (2002).

[12] P. Langacker and D. London, Phys. Rev. D 38, 886 (1988); E. Nardi, E. Roulet, and D. Tommasini, Phys. Lett. B 344, 225 (1995); T. C. Andre and J. L. Rosner, Phys. Rev. D 69, 035009 (2004); J. A. Aguilar-Saavedra, Phys. Lett. B 625, 234 (2005); 633, 792(E) (2006).

[13] M. Veltman, Nucl. Phys. B123, 89 (1977); Diagrammatica (Cambridge University Press, Cambridge, 1994), pp. 159161.

[14] J. C. Montero, F. Pisano, and V. Pleitez, Phys. Rev. D 47, 2918 (1993); H. Fanchiotti, C. Garcia-Canal, and W. A. Ponce, Europhys. Lett. 72, 733 (2005).

[15] F. Pisano and V. Pleitez, Phys. Rev. D 46, 410 (1992).

[16] S. L. Glashow and S. Weinberg, Phys. Rev. D 15, 1958 (1977).

[17] J. Hewett and T. Rizzo, Phys. Rep. 183, 193 (1989); F. del Aguila, Acta Phys. Pol. B 25, 1317 (1994); A. Leike, Phys. Rep. 317, 143 (1999); X-G. He and G. Valencia, Phys. Rev. D 68, 033011 (2003).

[18] H. Georgi and S. L. Glashow, Phys. Rev. D 7, 2457 (1973).

[19] X-G. He and G. Valencia, Phys. Rev. D 74, 013011 (2006).

[20] K. Cheung, C-W. Chiang, N. G. Deshpande, and J. Jiang, hep-ph/0604223. 